\title{
Gemcitabine plus nab-paclitaxel followed by maintenance treatment with gemcitabine alone as first-line treatment for older adults with locally advanced or metastatic pancreatic cancer
}

\author{
Roberto Petrioli a,*, Pamela Torre ${ }^{\mathrm{a}}$, Guido Pesola ${ }^{\mathrm{a}}$, Giovanni Paganini ${ }^{\mathrm{b}}$, Loretta Paolelli ${ }^{\mathrm{c}}$, \\ Salvatora Tindara Miano ${ }^{\mathrm{a}}$, Ignazio Martellucci ${ }^{\mathrm{a}}$, Guido Francini ${ }^{\mathrm{a}}$, Edoardo Francini ${ }^{\mathrm{d}, \mathrm{e}}$ \\ a Medical Oncology Unit, Department of Medicine, Surgery and Neurosciences, University of Siena, Italy \\ b General Medicines' Oncology, Pieve di Coriano Hospital, ASST Mantova, Italy \\ c Medical Oncology, Vito Fazzi Hospital, Lecce, Italy \\ d La Sapienza University, Rome, Italy \\ e Medical Oncology Unit, Misericordia Hospital, Grosseto, Italy
}

\section{A R T I C L E I N F O}

\section{Article history:}

Received 11 April 2019

Received in revised form 20 July 2019

Accepted 21 August 2019

Available online $\mathrm{xxxx}$

\section{Keywords:}

Pancreatic cancer

Gemcitabine

Nab-paclitaxel

Older adults

Maintenance

\begin{abstract}
A B S T R A C T
Objectives: The aim of this study was to evaluate the efficacy and safety of the combination Gemcitabine (Gem) plus nab-Paclitaxel (NabP) (Gem/NabP), followed by maintenance Gem in older adults with locally advanced or metastatic pancreatic cancer (PC).

Materials and methods: In this prospective observational study, the induction chemotherapy consisted of NabP $125 \mathrm{mg} / \mathrm{m}^{2}$ followed by Gem $1000 \mathrm{mg} / \mathrm{m}^{2}$ on days 1,8 , and 15 of a 4 -week cycle. After a maximum of 3 cycles, patients without evidence of progressive disease (PD) were administered Gem $1000 \mathrm{mg} / \mathrm{m} 2$ weekly for 3 of 4 weeks as maintenance therapy until documentation of PD or unacceptable toxicity. The primary endpoint was six-month disease-control rate (DCR).

Results: Overall, 36 patients $>70$ years with metastatic or locally advanced PC were enrolled at participating Institutions. After completion of Gem/NabP, 18 (50\%) patients achieved partial response, 13 (36\%) had stable disease, and 5 (14\%) had PD. Thirty-one patients (86\%) received Gem monotherapy as maintenance treatment for a median of 3 cycles (range, 2-9 cycles). Six-month DCR was 61\% (95\% CI, 45-77), median PFS was 6.4 months (95\% CI, 5.4-8.3), and median OS was 13.4 months (95\% CI, 11.1-16.7). During Gem/NabP regimen, the most common grade 3 toxicity included neutropenia (22\%), anemia (19\%) and thrombocytopenia (8\%). Grade 3 neuropathy was not observed. During Gem maintenance therapy, grade 3 hematological toxicity was described in 6 patients (19\%). Conclusion: Gem/NabP followed by maintenance Gem appears to be safe and effective for older patients with locally advanced or metastatic PC.
\end{abstract}

(c) 2019 Published by Elsevier Ltd.

\section{Introduction}

The incidence of pancreatic cancer (PC) in the United States is steadily rising and 55,440 new cases were expected for 2018 [1]. Most patients present with locally advanced unresectable or metastatic disease and the 5-year survival rate is approximately $3 \%$ mainly due to the early metastatic dissemination $[1,2]$.

Gemcitabine (Gem) has been the standard first-line treatment for patients with advanced PC until 2011 when the chemotherapy combination fluorouracil + irinotecan + oxaliplatin (FOLFIRINOX) was shown to increase median overall survival (OS) compared with

\footnotetext{
* Corresponding author at: Medical Oncology Unit, Department of Medicine, Surgery and Neurosciences, University of Siena, Viale Bracci, 53100 Siena, Italy.

E-mail address: r.petrioli@ao-siena.toscana.it (R. Petrioli).
}

Gem alone (11.0 vs.. 6.8 months; hazard ratio [HR], 0.57 ; 95\% confidence interval [CI], 0.45-0.73; $P<.001$ ) [3]. Despite these positive results, a higher rate of severe adverse events have been observed with this combination regimen and thus particular caution should be exercised when using it for older subjects [3].

In 2013, the international phase III randomized trial MPACT demonstrated that the combination gemcitabine plus nab-paclitaxel (Gem/ NabP) compared to Gem monotherapy for patients with metastatic PC and a Karnofsky performance status (KPS) $\geq 70$ prolongs median OS ( 8.5 vs. 6.7 months; $\mathrm{HR}=0.72 ; 95 \% \mathrm{CI}, 0.62-0.83 ; P<.001$ ), median progression free survival (PFS) (5.5 vs. 3.7 months; $\mathrm{HR}=0.69 ; 95 \% \mathrm{CI}$, $0.58-0.82 ; \mathrm{P}<.001)$, and objective response rate (ORR) (23\% vs. $7 \%$; $\mathrm{P}<.001$ ) [4]. The incidence of the most common grade $\geq 3$ treatment-related adverse events (neutropenia, fatigue, and peripheral neuropathy) was higher in the Gem/NabP group than in the Gem alone 
group. Particularly, grade 3 or higher peripheral neuropathy was reported in $17 \%$ vs $1 \%$ of the patients in the two groups. Of note, most patients receiving Gem/NabP were $<65$ years and had a good performance status (PS), hence safety and efficacy data for older and vulnerable patients are limited. Older adults with cancer often have comorbidities which complicate the administration of aggressive treatments. In clinical practice, Gem monotherapy or best supportive care are often recommended for patients $>75-80$ years with advanced PC [5]. On the other hand, older patients with good PS and considered fit according to comprehensive geriatric assessment (CGA) might benefit by the administration of a multidrug treatment. In colorectal cancer, stop and go or intermittent chemotherapy were shown to be valid alternative treatment schedules to reduce neuropathy [6-8]. However, to the best of our knowledge, current literature lacks data surrounding the use of similar treatment strategies with Gem/NabP in advanced PC. Given the relatively good activity and toxicity profile of Gem, the administration of Gem/NabP for a limited number of cycles followed by Gem as maintenance could minimize the severity of adverse events in the older adult.

The present study sought to prospectively evaluate the clinical efficacy and tolerability of induction chemotherapy with Gem/NabP followed by Gem as maintenance treatment for non-frail patients $>70$ years with advanced PC.

\section{Patients and methods}

\subsection{Eligibility criteria}

This multi-institution prospective observational study included patients $>70$ years with locally advanced or metastatic histologically or cytologically proven pancreatic cancer (PC), who had not previously received chemotherapy for this stage of disease. Any adjuvant treatment completed at least 12 months before inclusion was allowed. All patients had to present at study entry with an Eastern Cooperative Oncology Group (ECOG) PS of $\leq 2$ and adequate hematological (leukocytes $\geq 3000 / \mathrm{mm} 3$; hemoglobin $\geq 10 \mathrm{~g} / \mathrm{dL}$, platelets $\geq 100,000 / \mathrm{mm} 3$ ), renal (serum creatinine $\leq 2.0 \mathrm{mg} / \mathrm{dL}$ ) and hepatic function (serum bilirubin $\leq 2.0 \mathrm{mg} / \mathrm{dL}$ ). The exclusion criteria were a congestive heart failure, recent myocardial infarction, or co-existence of any other malignant disease except basal cell carcinoma of the skin. Patients $<75$ years with ECOG PS $=0$ were also excluded as considered candidate to receive conventional Gem/NabP or FOLFIRINOX chemotherapy.

Patients with high bilirubin and/or high aspartate and alanine aminotransferase levels were admitted provided that stenting or bypass surgery with the aim of stabilizing liver function were performed prior to starting chemotherapy. In this respect, total bilirubin, aspartate, or alanine aminotransferase levels $>3 \mathrm{x}$ the upper normal limit before starting treatment were also exclusion criteria. A baseline geriatric assessment including activities daily living (ADL), instrumental activities of daily living (IADL), and the Charlson score for comorbidities [9] was carried out, and patients were consequently classified as fit, vulnerable, and frail. Specifically, subjects with ADL limitations and more than two comorbidities were classified as frail and were excluded from the study [10]. All patients signed the informed consent to treatment. Patients were recruited from five participating institutions including our Medical Oncology Unit which designed and coordinated the study (Mantova, northern Italy; Siena, Grosseto, Rome, center Italy; Lecce, south Italy).

\subsection{Treatment plan}

Induction chemotherapy consisted of Nab-Paclitaxel $125 \mathrm{mg} / \mathrm{m}^{2}$ followed by Gem $1000 \mathrm{mg} / \mathrm{m}^{2}$ on days 1,8 , and 15 , every 4 weeks ( 1 cycle) for a maximum of 3 cycles. For patients $>80$ years, the number of planned cycles was two. Full-dose Gem and NabP was administered only to patients $\leq 80$ years and fit. Chemotherapy dose was reduced by $20-30 \%$ for vulnerable patients and subjects $>80$ years, to minimize the occurrence of treatment-related adverse events. Maintenance treatment consisted of weekly Gem $1000 \mathrm{mg} / \mathrm{m}^{2}$ ( $800 \mathrm{mg} / \mathrm{m}^{2}$ for vulnerable or $>80$ years patients) for 3 of 4 weeks and was continued until documentation of progressive disease or unacceptable toxicity. Cycles were administered if serum white blood cells count was $>3000 / \mathrm{mm}^{3}$, granulocytes $>1500 / \mathrm{mm}^{3}$, and platelets $>100,000 / \mathrm{mm}^{3}$.

\subsection{Patient evaluation}

All patients underwent a baseline ECG and their left ventricular ejection fraction (LVEF) was measured by means of a multiple gated acquisition scan or echocardiography. A complete physical examination, monitoring of symptoms and toxic effects, assessment of renal function, CEA and CA 19-9 markers, and a complete blood count were performed on patients at the start of every chemotherapy cycle. The disease was re-assessed after completion of three cycles of Gem/NabP, and then thereafter at 3-month intervals until there was evidence of disease progression (PD). Objective tumor response was evaluated according to Response Evaluation Criteria in Solid Tumors (RECIST criteria, version 1.1).

\subsection{Toxicity}

The common toxicity criteria of the National Cancer Institute Common Terminology Criteria for Adverse Events (NCI-CTCAE; version 4.02) were used to assess toxicity. Treatment was delayed if, on the planned day of therapy, the neutrophil count was $<1500 / \mathrm{mm}^{3}$, or the platelet count was $<100,000 / \mathrm{mm}^{3}$, or the patient had persistent diarrhea or stomatitis $>$ grade 1 . Any patient who required $>3$ weeks to recover from side effects was excluded from the study. A $25 \%$ dose reduction of the chemotherapy drugs was applied for subsequent courses in case of grade 4 hematologic or any $\geq$ grade 3 organ toxicity.

\subsection{Statistical considerations}

The primary endpoint of the study was 6-months disease-control rate (DCR) defined as the proportion of patients who obtained stable disease (SD) or partial (PR) or complete response (CR) within 6 months after starting induction treatment. Most studies investigating Gem monotherapy for older adults with advanced PC report that approximately $20-25 \%$ of patients are still progression-free at 6 months after treatment onset $[5,11]$. The hypothesis of the current study was that, using Gem/NabP as induction chemotherapy and Gem as maintenance treatment, at least $40 \%$ of patients would be progression-free after 6 months from chemotherapy initiation. It was calculated that at least 33 patients should be enrolled to yield a $80 \%$ probability to correctly select the therapy when it is superior by absolute difference of $20 \%$ in 6-months DCR (Simon's minimax design) [12]. Safety, PFS (measured as the time from the first chemotherapy to PD or death from any cause or last follow-up visit, whichever came first), and OS (assessed from treatment start to death or last follow-up visit, whichever came first) and ORR (as the rate of patients achieving PR or CR) were the secondary endpoints. Kaplan-Meier method was utilized to assess the distributions of PFS and OS, including median time-to-event and its 95\% CI. Statistical analyses were performed by MEDCALC software.

\section{Results}

\subsection{Patient characteristics}

Between May 2014 and June 2018, 36 patients with advanced or metastatic PC were treated with Gem/NabP and maintenance Gem at participating institutions. Baseline demographic and clinical characteristics of patients are described in Table 1 . Median age was 77 years (range, 71-86 years) and 6 of 36 patients (17\%) were aged over 80 years. Most patients ( 33 of 36; 92\%) had an ECOG PS of 0 or 1 and 21 of 36 patients (58\%) were classified as fit. Six patients (17\%) had undergone surgical resection of the primary tumor with curative intent 
Table 1

Main patient characteristics at baseline.

\begin{tabular}{ll}
\hline Variable & All patients $(N=36)$ \\
\hline Age, years (median, range) & $77(71-86)$ \\
$>75$ & $21(58)$ \\
$>80$ & $6(17)$ \\
Sex & \\
Male & $24(67)$ \\
Female & $12(38)$ \\
ECOG performance status & $16(45)$ \\
0 & $17(47)$ \\
1 & $3(8)$ \\
2 & \\
Previous pancreatic surgery & $28(78)$ \\
No & $6(17)$ \\
Yes & \\
CA 19.9 & $31(86)$ \\
Abnormal & $5(14)$ \\
Normal & $851.7(2.6-21,360.4)$ \\
Median, U/mL (range) & \\
Geriatric assessment, No (\%) & $21(58)$ \\
Fit & $15(42)$ \\
Vulnerable & $1(0-2)$ \\
Charlson Comorbidity Index, No (\%) & \\
Median (range) & $32(89)$ \\
Adjuvant chemotherapy & $4(11)$ \\
None & $8(22)$ \\
Yes & $28(78)$ \\
Locally unresectable & $21(58)$ \\
Metastasized & $27(75)$ \\
Sites of metastases & $11(31)$ \\
Liver & \\
Lymph node & \\
Lung & \\
Peritoneal & \\
Number of metastatic sites & \\
$>1$ & \\
\hline
\end{tabular}

Note: Data are expressed as numbers (\%) except where otherwise noted.

and were offered to enter the study once the metastatic disease was radiologically proven. Twenty-eight of 36 patients (78\%) presented at diagnosis with distant disease and twenty-one (58\%) had liver metastases. At baseline, the majority of patients ( 22 of $36 ; 61 \%$ ) had $>1$ comorbidity (Table 2) and the most frequent were cardiovascular diseases (16 of 36 ; $44 \%)$. Polypharmacy $(5+)$ was documented in $38.8 \%$ of patients. $\mathrm{ADL}$ and instrumental ADL dependencies were reported in $27.7 \%$ and $38.8 \%$ of patients, respectively. The most affected basic ADLs were walking, transfers and eating. Concerning IADLs, household management was most commonly affected. Weight loss was described in $58.3 \%$ of patients, and the mean value of baseline body mass index was $25.4+5$ standard deviation. Cognitive impairment was reported in 5 patients (13.8\%).

\subsection{Efficacy}

All 36 patients completed the planned chemotherapy and were evaluable for response and toxicity. A total of 102 cycles of Gem/NabP (median, 3 cycles; range $2-3$ ) was administered. The median relative

Table 2

Patient comorbidities.

\begin{tabular}{ll}
\hline Comorbidities & Number of patients (\%) \\
\hline Cardiovascular & $17(47)$ \\
Hypertension & $14(39)$ \\
Coronary artery disease & $6(17)$ \\
Arrhythmia & $4(11)$ \\
Diabetes mellitus & $9(25)$ \\
Dyslipidemia & $6(17)$ \\
Respiratory & $8(22)$ \\
Genitourinary & $5(14)$ \\
$>1$ comorbidity & $22(61)$ \\
\hline
\end{tabular}

Table 3

Results.

\begin{tabular}{ll}
\hline Variables & \\
\hline Objective response rate, N (\%) & $18(50)$ \\
Complete response & 0 \\
Partial response & $18(50)$ \\
Stable disease & $13(36)$ \\
Progressive disease & $5(14)$ \\
6-month disease control rate, \% (95\% CI) & $61(45-77)$ \\
Median progression free survival, months (95\% CI) & $6.4(5.4-8.3)$ \\
Median overall survival, months (95\% CI) & $13.4(11.1-16.7)$ \\
Median follow-up, months (range) & $15.3(5-42)$ \\
\hline
\end{tabular}

dose intensity (RDI) was $88 \%$ for Gem and $86 \%$ for NabP. Six patients $>80$ years received two cycles of induction chemotherapy. The starting dose of NabP and GEM was $125 \mathrm{mg} / \mathrm{m}^{2}$ and $1000 \mathrm{mg} / \mathrm{m}^{2}$, respectively, in 19 fit patients $\leq 80$ years and $100 \mathrm{mg} / \mathrm{m} 2$ and $800 \mathrm{mg} / \mathrm{m} 2$, respectively, in 15 vulnerable patients and in 2 fit patients $>80$ years. The ORR was $50 \%$. No patient achieved CR while 18 subjects (50\%) achieved PR and 13 (36\%) SD, as best response to therapy after initial Gem/NabP (Table 3). The 31 patients (86\%) who achieved PR or SD after induction treatment received maintenance chemotherapy with Gem for a median of 3 cycles (range, 2-9 cycles). The ORR for Gem maintenance treatment was $6 \%$. Twenty-nine patients discontinued maintenance chemotherapy because of PD and for 8 of these, fit and $<80$ years, NabP was reintroduced. One patient was lost to follow-up after seven months from the onset of induction chemotherapy while two patients remain on maintenance treatment at the time of this writing. The 6-months DCR was 61\% (95\% CI, 45-77) and median PFS was 6.4 months (95\% CI, 5.4-8.3). After a median follow-up of 15.3 months (range, 5-42 months), a total of 31 patients were deceased and median OS was 13.4 months (95\% CI 11.1-16.7). Distributions of PFS and OS for the 36 enrolled patients are described in Fig. 1. In the locally advanced and metastatic subgroups, PFS was 8.3 months and 6.1 months and OS 17.3 and 11.2 months, respectively. A relevant CA 19-9 response, defined as a decrease $>50 \%$ from an elevated baseline value, was observed in 27 (75\%) patients after induction chemotherapy. Finally, 16 patients (44\%) received an irinotecan-based chemotherapy as second-line treatment and 9 (25\%) patients received capecitabine alone as third-line therapy.

\subsection{Toxicity}

Adverse events rates during Gem/NabP induction regimen are summarized in Table 4. Grade 3 toxicity included neutropenia (22\%), anemia

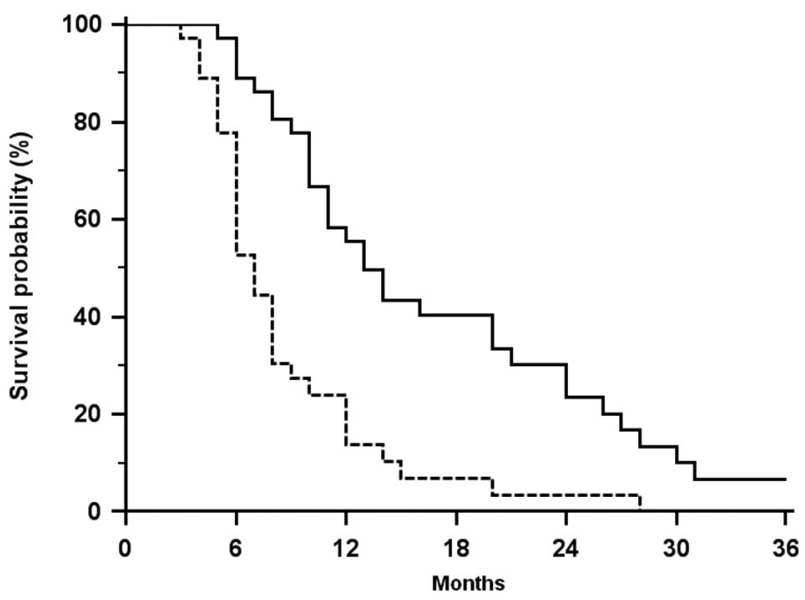

Fig. 1. Kaplan-Meier estimates of PFS (-) and OS (-) from Gem/NabP start. Abbreviations: Gem/NabP: gemcitabine plus nab-paclitaxel; PFS: progression-free survival; OS: overall survival. 
Table 4

Adverse events during gemcitabine plus nab-paclitaxel induction treatment.

\begin{tabular}{|c|c|c|c|}
\hline & Grade 2 & Grade 3 & Grade 4 \\
\hline \multicolumn{4}{|l|}{ Hematological } \\
\hline Neutropenia & $5(14)$ & $8(22)$ & $2(5)$ \\
\hline Anemia & $13(36)$ & $7(19)$ & 0 \\
\hline Trombocytopenia & $6(17)$ & $3(8)$ & 0 \\
\hline \multicolumn{4}{|l|}{ Non-hematological } \\
\hline Nausea & $6(17)$ & 0 & 0 \\
\hline Vomiting & $4(11)$ & 0 & 0 \\
\hline Fatigue & $8(22)$ & $1(3)$ & 0 \\
\hline Stomatitis & $3(8)$ & 0 & 0 \\
\hline Sensory neuropathy & $6(17)$ & 0 & 0 \\
\hline Diarrhea & $3(8)$ & $2(5)$ & 0 \\
\hline Constipation & $4(11)$ & $2(5)$ & 0 \\
\hline
\end{tabular}

Note: Data are expressed as numbers (\%) except where otherwise noted.

(19\%), thrombocytopenia (8\%). Sensory neuropathy, related to the use of NabP, never reached grade 3. Due to side effects, a total of $12 \mathrm{Gem} /$ NabP administrations were delayed and a dose reduction of GEM/ NabP was necessary for 9 (25\%) patients. Maintenance treatment was associated with mild toxicity and only 6 patients (19\%) experienced grade 3 hematologic toxicity (Table 5 ). Six patients (17\%) needed a dose reduction of Gem monotherapy.

\section{Discussion}

The combination treatment Gem/NabP improved median OS, PFS, and ORR compared to Gem alone for patients with metastatic PC in a large randomized phase 3 trial; however, data on the efficacy and safety of this doublet chemotherapy for older adults with advanced PC are currently limited and, also considering that older patients frequently present with comorbidities, mono-chemotherapy or best supportive care are generally preferred in routine clinical care. A reasonable approach for non-frail older adults with advanced PC could be an initial shortterm combination chemotherapy followed by Gem alone as maintenance treatment. In this respect, to the best of our knowledge, this is the first study suggesting the feasibility and activity of Gem/NabP for 3 cycles followed by Gem monotherapy as maintenance treatment for patients over 70 years with locally advanced or metastatic PC. Despite the limitations of a small study, the 61\% 6-month DCR, 6.4 months median PFS, and 13.4 months median OS achieved in our analysis are in line with the results commonly observed with more active combination chemotherapies in younger populations $[3,4,13]$. In this respect, a recent multicenter real-world study of 75 patients treated with Gem/NabP for advanced PC reported similar or slightly shorter median PFS (5.2 vs. 6.4 months, respectively) and OS (10.9 vs. 13.4 months, respectively) than those observed in the current study [14]. Of note, although the above-mentioned retrospective experience confirmed the effectiveness and safety of first-line Gem/NabP in a real world setting, grade 3-4 hematologic toxicity occurred frequently and dose reductions were needed in a higher proportion of patients compared to our report ( $80 \%$ vs. $25 \%$ ). A recent phase III study analysing NabP plus the oral fluoropyrimidine derivative $\mathrm{S}-1$ followed by $\mathrm{S}-1$ as maintenance

Table 5

Adverse events during gemcitabine maintenance treatment.

\begin{tabular}{lll}
\hline & Grade 2 & Grade 3 \\
\hline Neutropenia & $12(39)$ & $4(13)$ \\
Anemia & $21(68)$ & $6(19)$ \\
Trombocytopenia & $15(48)$ & $6(19)$ \\
Nausea/vomiting & $2(6)$ & 0 \\
Fatigue & $4(13)$ & $1(3)$ \\
Diarrhea & $1(3)$ & 0 \\
Rash & $1(3)$ & 0 \\
\hline
\end{tabular}

Note: Data are expressed as numbers (\%) except where otherwise noted. treatment for advanced PC patients reported results in terms of ORR (53.1\%), median PFS (6.2 months), and OS (13.6 months) similar to our analysis [15]. However, the median age of the study population was much younger (53 vs. 77 years) compared to our report and only 6 patients were over 80 years old. Conversely, a retrospective multicenter study of unselected PC patients treated with Gem/NabP described lower median PFS (5.0 months) and OS (7.2 months) compared with our findings. However, it should be noted that this study population had poorer ECOG PS and the median RDI was only 66.7\% [16]. In fact, the authors reported Gem/NabP dose reductions or discontinuations for $65 \%$ and $17 \%$ of patients, respectively, due to toxicity. A point to consider is that the current prospective observational study included a few patients ( 8 of $36 ; 22 \%$ ) with locally advanced unresectable tumor. This stage of PC is usually associated with a better prognosis compared to metastatic disease [17]. As a matter of fact, despite the subgroups being quite small, a considerably higher median OS could be observed for patients with advanced PC vs. distant disease (18.3 vs. 11.2 months, respectively). Other authors recently reported similar outcomes for locally advanced or metastatic PC patients cohort treated with Gem/NabP or FOLFIRINOX [18].

Interestingly, alternative treatment strategies were recently experimented to minimize Gem/NabP adverse events. A "stop and go" approach (named OPTINAB), consisting of suspending NabP at first occurrence of grade 3 neuropathy and re-instituting it at time of biochemical or radiological progression, was recently applied in a small retrospective clinical study including advanced $P C$ patients receiving Gem/NabP. The OPTINAB strategy was well tolerated and achieved an OS (11.7 months; range, 9.5-17 months) comparable to that of our analysis which, however, included older patients and mostly (78\%) at a later stage of disease [19]. Furthermore, our results compare well with those recently reported with a less intensive regimen of Gem/NabP such as a biweekly schedule (about 5 months median PFS and 10 months OS) $[20,21]$. However, although older adults participated in the aforementioned studies, the median age of enrolled patients was at most 67 years, while the median age was 77 years in our population study.

Despite the advanced median age and considerable rate of patients with at least 2 comorbidities of our study population, treatment was well tolerated and there were no unexpected toxic effects. The treatment strategy we proposed seemed to allow for avoiding the cumulative toxicities of NabP and prolong duration of chemotherapy with GEM alone as maintenance treatment. Of note, in several cases the starting dose of Gem and NabP was lower than the conventional starting dose of $1000 \mathrm{mg} / \mathrm{m} 2$ and $125 \mathrm{mg} / \mathrm{m} 2$, respectively. However, such dose reductions were due to the high proportions of subjects classified as non-fit (42\%) or presenting with $>1$ concomitant disease $(61 \%)$. This could also partly explain the relatively low rate of grade $3-4$ neutropenia (22\%) and grade 2 only nausea and vomiting observed in our study during initial Gem/NabP regimen. A similar or also higher grade 3-4 hematologic toxicity (up to $40 \%$ ) compared with results observed in our population study was reported with the modified biweekly Gem/NabP regimen $[20,21]$. Nevertheless, despite the biweekly Gem/NabP regimen appearing to be effective with an acceptable toxicity profile in advanced PC patients, its potential benefits in older adults in comparison with the standard weekly regimen needs to be confirmed by prospective studies.

In addition, it should be noted that sensory neuropathy never reached grade 3 . This safety profile is better than usually reported with conventional Gem/NabP for younger patients and could be mainly the result of the shorter exposure to NabP achieved with our treatment schedule, the accurate selection of non-frail subjects, and the Gem and NabP starting dose reductions. In this regard, despite still not existing consensus over the definition of frailty status, we deem that an age $<85$ years, $<3$ concomitant comorbidities, the ability to perform the daily living activities, and no cognitive impairment are the elements which best define a non-frail patient.

A baseline geriatric evaluation is useful to decide if monochemotherapy or a more aggressive treatment will be used in older adults with advanced 
PC. In this setting the ongoing GrantPax study is the first trial worldwide evaluating a CGA-driven treatment allocation to personalize cancer therapy for older adults with metastatic pancreatic ductal adenocarcinoma [22].

The one-arm design, relatively small population, and long study duration (4 years) are potential limits that prevent us from drawing general conclusions from our report. Although careful monitoring of potential side effects in older patients is recommended, the current study suggests that induction chemotherapy with Gem/NabP followed by maintenance treatment with Gem monotherapy might be safely proposed as first-line treatment for non-frail patients aged $>70$ years with advanced PC. Despite larger randomized trials are required to confirm our results, the present findings could aid treatment decision making for non-frail older adults with locally advanced or metastatic PC.

\section{Author contribution}

Roberto Petrioli: design and writing.

Pamela Torre: data collection and writing.

Guido Pesola: data collection.

Giovanni Paganini: patient enrollment.

Loretta Paolelli: patient enrollment.

Salvatora Tindara Miano: data analysis.

Ignazio Martellucci: data analysis.

Guido Francini: design and writing.

Edoardo Francini: writing and patient enrollment.

\section{Declaration of Competing Interest}

All the authors declare that no potential or actual conflicts of interest exist.

\section{References}

[1] Siegel RL, Miller KD, Jemal A, et al. Cancer statistics, 2018. CA Cancer J Clin 2018;68: 7-30.

[2] Vincent A, Herman J, Schulick R, Hruban RH, Goggins M. Pancreatic cancer. Lancet 2011;378:607-20.

[3] Conroy T, Desseigne F, Ychou M, Bouché O, Guimbaud R, Bécouarn Y, et al. FOLFIRINOX versus gemcitabine for metastatic pancreatic cancer. N Engl J Med 2011;364:1817-25.

[4] Von Hoff DD, Ervin T, Arena FP, Chiorean EG, Infante J, Moore M, et al. Increased survival in pancreatic cancer with nab-paclitaxel plus gemcitabine. N Engl Med 2013; 369:1691-703.

[5] Hidalgo M. Pancreatic cancer. N Engl J Med 2010;362:1605-17.
[6] Petrioli R, Paolelli L, Marsili S, Civitelli S, Francini E, Cioppa T, et al. FOLFOX-4 stop and go and capecitabine maintenance chemotherapy in the treatment of metastatic colorectal cancer. Oncology 2006;70:345-50.

[7] Vaidyanathan G, Groman A, Wilding G, Fakih MG. Stop and go FOLFOX plus bevacizumab chemotherapy in the first-line treatment of metastatic colorectal cancer. Oncology 2010;79:67-71.

[8] Díaz-Rubio E, Gómez-España A, Massutí B, Sastre J, Abad A, Valladares M, et al. Group for the Treatment of digestive Tumors. First-line XELOX plus bevacizumab followed by XELOX plus bevacizumab or single-agent bevacizumab as maintenance therapy in patients with metastatic colorectal cancer: the phase III MACRO TTD study. Oncologist 2012;17:15-25.

[9] Repetto L, Fratino L, Audisio RA, Venturino A, Gianni W, Vercelli M, et al. Comprehensive geriatric assessment adds information to eastern cooperative oncology group performance status in elderly cancer patients: an Italian Group for Geriatric Oncology Study. J Clin Oncol 2002;20:494-502.

[10] Balducci L, Extermann M. Management of cancer in the older person: a practical approach. Oncologist 2000;5:224-37.

[11] Colucci G, Labianca R, Di Costanzo F, Gebbia V, Carteni G, Massidda B, et al. Randomized phase III trial of gemcitabine plus cisplatin compared with single-agent gemcitabine as first-line treatment of patients with advanced pancreatic cancer: the GIP-1 study. J Clin Oncol 2010;28:1645-51.

[12] Simon R. Optimal two-stage designs for phase II clinical trials. Control Clin Trials 1989;10:1-10.

[13] De Vita F, Ventriglia J, Febbraro A, Laterza MM, Fabozzi A, Savastano B, et al. NABpaclitaxel and gemcitabine in metastatic pancreatic ductal adenocarcinoma (PDAC): from clinical trials to clinical practice. BMC Cancer 2016;16(709):1-8.

[14] Blomstrand H, Scheibling U, Bratthäll C, Green H, Elander NO. Real world evidence on gemcitabine and nab-paclitaxel combination chemotherapy in advanced pancreatic cancer. BMC Cancer 2019 Jan 8;19(40):1-9.

[15] Zhang W, Du C, Sun Y, Yang L, Cui C, Jiang Z, et al. Nab-paclitaxel plus S-1 as first-line followed by S-1 maintenance for advanced pancreatic adenocarcinoma: a singlearm phase II trial. Cancer Chemother Pharmacol 2018;82:655-60.

[16] Fernández Ana, Salgado Mercedes, García Adelaida, Buxò Elvira, Vera Ruth, Adeva Jorge, et al. Prognostic factors for survival with nab-paclitaxel plus gemcitabine in metastatic pancreatic cancer in real-life practice: the ANICE-PaC study. BMC Cancer 2018; 18:1185:1-11.

[17] Philip PA, Mooney M, Jaffe D, Eckhardt G, Moore M, Meropol N, et al. Consensus report of the national cancer istitute clinical trials planning meeting on pancreas cancer treatment. J Clin Oncol 2009;27:5660-9.

[18] Vogl UM, Andalibi H, Klaus A, Vormittag L, Schima W, Heinrich B, et al. Nabpaclitaxel and gemcitabine or FOLFIRINOX as first-line treatment in patients with unresectable adenocarcinoma of the pancreas: does sequence matter? BMC Cancer 2019;19:28:1-8.

[19] Relias V, Maloney A, Smith MH, Saif MW. Does "OPTINAB" strategy ("stop-and-go") work in treatment of advanced pancreatic cancer (APC) with nab-paclitaxelgemcitabine? Cancer Chemother Pharmacol 2017;80:371-5.

[20] Ahn DH, Krishna K, Blazer M, Reardon J, Wei L, Wu C, et al. A modified regimen of biweekly gemcitabine and nab-paclitaxel in patients with metastatic pancreatic cancer is both tolerable and effective: a retrospective analysis. Ther Adv Med Oncol 2017;9:75-82

[21] Kokkali S, Tripodaki E-S, Drizou M, Stefanou D, Magou E, Zylis D, et al. Biweekly gemcitabine/nab-paclitaxel as first-line treatment for advanced pancreatic cancer. In Vivo 2018;32:653-7.

[22] Betge J, Chi-Kern J, Schulte N, Belle S, Gutting T, Burgermeister E, et al. A multicenter phase 4 geriatric assessment directed trial to evaluate gemcitabine $+/-$ nabpaclitaxel in elderly pancreatic cancer patients (GrantPax). BMC Cancer 2018;18:747. 